\title{
Das Leitbild des „europäischen Juristen“ - Gedanken zur Juristenausbildung und zur Rechtskultur in Deutschland"
}

\author{
Andreas Voßkuhle**
}

\section{A. Einleitung: Das Leitbild des Juristen im Wandel}

Im Jahr 1905 schrieb der junge Freiburger Privatdozent Hermann Kantorowicz:

\begin{abstract}
„Die herrschende Idealvorstellung vom Juristen ist die: Ein höherer Staatsbeamter mit akademischer Ausbildung, sitzt er, bewaffnet bloß mit einer Denkmaschine, freilich einer von der feinsten Art, in seiner Zelle. Ihr einziges Mobiliar ein grüner Tisch, auf dem das staatliche Gesetzbuch vor ihm liegt. Man reicht ihm einen beliebigen Fall, einen wirklichen oder nur erdachten, und entsprechend seiner Pflicht, ist er imstande, mit Hülfe rein logischer Operationen und einer nur ihm verständlichen Geheimtechnik, die vom Gesetzgeber vorherbestimmte Entscheidung im Gesetzbuch mit absoluter Exaktheit nachzuweisen". 1
\end{abstract}

Mit diesen Worten beginnt Kantorowicz seine provokante Streitschrift mit dem Titel „Der Kampf um die Rechtswissenschaft“. Das Bemerkenswerte an ihnen ist dabei nicht nur die darin zum Ausdruck kommende Leidenschaft, mit der schon vor mehr als hundert Jahren über das Leitbild des Juristen gestritten wurde. Interessant ist auch die Tatsache, dass Kantorowicz sein berühmtes Pamphlet ausgerechnet während eines Studienaufenthalts in Bologna ${ }^{2}$ verfasst hat. Dieser Umstand belegt nicht nur die Mobilität eines „,europäischen Juristen “ vor mehr als hundert Jahren. ${ }^{3}$ Nein, die Erwähnung des Namens „Bologna“ weckt darüber hinaus vielfältige Assoziationen. Denn Bologna ist wohl mehr als jede andere Stadt geeignet, die Tradition und Größe der Wurzeln europäischer Juristenausbildung zu versinnbildlichen, die im Bologna

* Bei dem Beitrag handelt es sich um die redigierte und mit Fußnoten versehene Fassung meines Festvortrags zur Eröffnung des 16. Deutschen Verwaltungsgerichtstag in Freiburg am 5. Mai 2010. Für die große Unterstützung bei der Erarbeitung des Beitrags danke ich meiner Wissenschaftlichen Mitarbeiterin am Bundesverfassungsgericht Frau RiVG Dr. Sigrid Emmenegger sehr herzlich.

* Prof. Dr. Andreas Voßkuhle ist Präsident des Bundesverfassungsgerichts und Direktor des Instituts für Staatswissenschaft und Rechtsphilosophie an der Albert-Ludwigs-Universität Freiburg.

1 Gnaeus Flavius (d.i., Hermann Kantorowicz), Der Kampf um die Rechtswissenschaft, Heidelberg 1906, S. 7.

2 Vgl. F. Kantorowicz Carter, Gustav Radbruch and Hermann Kantorowicz: To Friends and a Book Reflections on Gnaeus Flavius' Der Kampf um die Rechtswissenschaft (1906), German Law Journal 2007, 657 (670).

3 K. war in Posen geboren, hatte in Berlin und München studiert, wurde in Heidelberg promoviert und habilitierte sich in Freiburg i.Br. Seine Berufung auf eine Professur in Kiel erfolgte erst 1929; 1933 wurde er als einer der ersten jüdischen Rechtswissenschaftler vertrieben und emigrierte zunächst nach New York, dann Cambridge, vgl. J. Schröder, in: G. Kleinheyer/J. Schröder, Deutsche und Europäische Juristen aus neun Jahrhunderten, 5. Aufl., Heidelberg 2008, S. 237 ff.; G. Brender, Artikel „Kantorowicz, Hermann Ulrich“, in: M. Stolleis (Hrsg.), Juristen: Ein biographisches Lexikon, München 2001, S. 347 ff. (348). 
des 12. Jahrhunderts begründet wurde: ${ }^{4}$ Ohne Bologna wären das moderne europäische System der akademischen Ausbildung an Universitäten und die vom römischen Recht geprägte Rechtskultur nicht denkbar. ${ }^{5}$ Zugleich steht der Name im heutigen Kontext stellvertretend für aktuelle Diskussionen über die Reform der Juristenausbildung aus Anlass der sogenannten „Bologna-Erklärung“ von 29 europäischen Bildungsministern am 19. Juni 1999, deren Ziel die Harmonisierung von Studienbedingungen und die bessere Vergleichbarkeit von Hochschulabschlüssen ist. ${ }^{6}$

Die Frage, was einen guten Juristen ausmacht, ist in der Tat heute ebenso drängend wie im 12. und 13. Jahrhundert (der Glanzzeit Bolognas) oder im Jahr 1905 (dem Jahr, in dem Kantorowicz sein Pamphlet verfasste). Das gleiche gilt für die sich daran anschließende Frage, wie man zu einem „guten Juristen“ werden kann, ${ }^{7}$ welches Leitbild wir also der Ausbildung zugrundelegen. ${ }^{8}$ Nicht erst seit der Vereinheitlichung der Juristenausbildung im Deutschen Reich mit den Reichsjustizgesetzen von 1877 wird um ihre Inhalte und ihre Form gestritten. ${ }^{9}$ Die Diskussion steht augenfällig

4 H. Wieling, Juristenausbildung im Mittelalter, in: C. Baldus/T. Finkenauer/T. Rüfner (Hrsg.), Juristenausbildung in Europa zwischen Tradition und Reform, Tübingen 2008, S. 47 ff. (52): Die Glossatoren als die „Väter der europäischen Rechtswissenschaft“. Vgl. in diesem Zusammenhang bereits $R$. Böttcher, Der europäische Jurist, JöR n.F. 49 (2001), S. 1 ff.: „Es gab ihn einmal, den europäischen Juristen. Im 12. und 13. Jahrhundert strömten Studenten aus ganz Europa nach Bologna, um das wieder entdeckte römische Recht zu studieren ... Kehrten die Scholaren in ihre Heimatländer zurück, brachten sie eine neue Art des Denkens mit und in der Folge auch eine neue Identität (...) Es fand ein europäischer Wettbewerb der Rechtsfakultäten statt“; H. Prütting, Folgen der Globalisierung für die Juristenausbildung, Vortrag, 2005, abrufbar unter www.tokyo-jura-kongress2005.de/_documents/pruetting_de.pdf (Zugriff vom 22.3.2010), S. 2: „Der mittelalterliche Rechtsunterricht hatte bereits weitgehend globale Tendenzen“; J. Fried, Die Entstehung des Juristenstandes im 12. Jahrhundert - zur sozialen Stellung gelehrter Juristen in Bologna und Modena, Köln/Wien 1974.

5 Finkenauer/Rüfner, Einleitung, in: dies./Baldus (Hrsg.), Juristenausbildung (Fn. 4), S. 1.

6 Der Europäische Hochschulraum. Gemeinsame Erklärung der Europäischen Bildungsminister, 19. Juni 1999, Bologna. Abrufbar unter http://www.bmbf.de/pub/bologna_deu.pdf (Zugriff vom 1.4.2010). Allgemeine Informationen über den Stand der Umsetzung unter http://www.hrk.de (Zugriff vom 6.4.2010). Aus der mittlerweile fast uferlosen Literatur zu den Auswirkungen des „Bologna-Prozesses“ auf die Juristenausbildung vgl. nur T. Pfeiffer, Probleme alla bolognese: Juristenausbildung und Bologna, in: RW 1 (2010), S. 104 ff.; H. Konzen, Bologna-Prozess und Juristenausbildung, JZ 2010, S. 241 ff., sowie die rechtsvergleichenden Beiträge in: Baldus/ Finkenauer/Rüfner (Hrsg.), Juristenausbildung (Fn. 4).

7 Zur Frage nach dem „guten Richter“ vgl. jüngst M. Eckertz-Höfer, „Vom guten Richter“ - Ethos, Unabhängigkeit, Professionalität, DÖV 2009, S. 729 ff.

8 Vgl. zum „,europäischen Juristen“ bereits P. Häberle, Der Europäische Jurist, Zürich 2002; ders., Der europäische Jurist, JöR n.F. 50 (2002), S. 123 ff.; dazu I. Pernice, Peter Häberle: Der Europäische Jurist, in: A. Blankenagel/I. Pernice/H. Schulze-Fielitz (Hrsg.), Verfassung im Diskurs der Welt: Liber Amicorum für Peter Häberle zum siebzigsten Geburtstag, Tübingen 2004, S. 3 ff.; Böttcher, Jurist (Fn. 4), S. 1 ff.; zur Frage nach dem Leitbild vgl. auch M. Stolleis, Gesucht: Ein Leitbild der Juristenausbildung, NJW 2001, 200 ff.; zum Begriff des Leitbildes S. Baer, Schlüsselbegriffe, Typen und Leitbilder als Erkenntnismittel und ihr Verhältnis zur Dogmatik, in: E. Schmidt-Aßmann/W. Hoffmann-Riem (Hrsg.), Methoden der Verwaltungsrechtswissenschaft, Baden-Baden 2004, S. 223 ff. (232 ff.), auch zur Gefahr von Leitbildern ebd., (234). Nach der Abgrenzung von S. Baer (236 ff.) dürfte es sich bei dem im Folgenden entworfenen Konzept des „europäischen Juristen“ genau genommen wohl eher um ein Modell, nicht um ein „Leit-Bild“ handeln.

9 B. Pieroth, Literarische Streifzüge durch die Geschichte der Juristenausbildung in Deutschland, in: G. H. Gornig/U. Kramer/U. Volkmann (Hrsg.), Staat - Wirtschaft - Gemeinde: FS W. Frotscher zum 70. Geburtstag, Berlin 2007, S. 795 ff. (795). 
in einem engen Zusammenhang mit den zentralen wirtschaftlichen, sozialen und technischen Veränderungen innerhalb der Gesellschaft oder jedenfalls mit einem entsprechenden Wahrnehmungswandel. ${ }^{10}$ Denn es sind diese Veränderungen, auf die das Rechtssystem reagiert, und die ihrerseits zugleich durch Recht gestaltet oder gar erst erzeugt werden. Die Berufung des Juristen ist es, diese Prozesse des Wandels des Rechts und des Wandels durch Recht aktiv zu begleiten Das hat auch Auswirkungen auf das Anforderungsprofil juristischer Tätigkeit. Indem wir immer wieder erneut über die Ausbildung des Juristen nachdenken, vergewissern wir uns daher gleichzeitig über seine Aufgabe in der heutigen Zeit und den Zustand des Rechtssystems.

\section{B. Einwirkungskräfte auf das Selbstverständnis und die Tätigkeitsfelder des Juristen}

Werfen wir zunächst einen Blick auf einige zentrale gegenwärtige Einwirkungskräfte auf das Selbstverständnis und die Tätigkeitsfelder des Juristen.

\section{Europäisierung}

An erster Stelle ist hier die allgegenwärtige „Europäisierung“ von Recht, Staat und Gesellschaft zu nennen. ${ }^{11}$ Im Kaiserreich war der idealtypische Jurist - wie Kantorowicz ihn überzeichnet rekonstruiert, um ihn sodann mit spitzer Feder zu bekämpfen ${ }^{12}$ - als „Staatsdiener" auf den Nationalstaat und dessen Recht fixiert. ${ }^{13}$ In einer Zeit, in der „offene Staatlichkeit“ zur „Überlebensvoraussetzung“ des Staates geworden ist $\mathrm{t}^{14}$, erweist sich diese Perspektive als entschieden zu eng.

Als Mitgliedstaat der Europäischen Union ist die Bundesrepublik Deutschland fest eingebunden in ein komplexes europäisches Mehrebenensystem. Wir sprechen in

10 Vgl. Stolleis, Leitbild (Fn. 8), S. 200: „Der Alltag der Juristenausbildung deutet auf Kontinuität (...) Aber dieser Eindruck täuscht. Der gesamte Kontext der Juristenausbildung ändert sich (...).

11 Vgl. auch A. v. Bogdandy, Beobachtungen zur Wissenschaft vom Europarecht, Der Staat 40 (2001), S. 3 ff. (43) Fn. 208: Europäisierung der Juristenausbildung als Voraussetzung einer Europäisierung der nationalen Rechtssysteme.

12 Vgl. zur Tradition solcher „Rechtskritik“ im Sinne einer „Juristenkritik“ jüngst J. Benedict, Rechtskritik, RTh 40 (2009), S. 337 ff. (345 ff.) (a]"Richterschelte“; b] „Advokatenschelte“); s. für die unterschiedlichen Bedeutungsgehalte des Ausdrucks „Rechtskritik“ S. Emmenegger, Gesetzgebungskunst, Tübingen 2006, S. 110.

13 Vgl. - unter Verweis auf Bleek, Von der Kameralausbildung zum Juristenprivileg: Studium, Prüfung und Ausbildung der höheren Beamten des allgemeinen Verwaltungsdienstes in Deutschland im 18. und 19. Jahrhundert, Berlin 1972 -: Pieroth, Literarische Streifzüge (Fn. 9), S. 797: Erst im 18. Jahrhundert beginne die Phase, in der die Juristenausbildung zu einem staatlichen Regelungsobjekt werde. Die deutschen Territorialstaaten hätten dabei das Ziel verfolgt, ihre Herrschaft durch eine leistungsfähige Staatsdienerschaft auszubauen; dabei bedienten sie sich vornehmlich juristisch Vorgebildeter und begründeten das so genannte Juristenmonopol.

14 R. Wahl, Internationalisierung des Staates, in: ders., Verfassungsstaat, Europäisierung, Internationalisierung, Frankfurt am Main 2003, S. 17 ff. (17) im Anschluss an A. Hollerbach, Globale Perspektiven der Rechts- und Staatsentwicklung, Freiburger Universitätsblätter, Heft 111, März 1991, S. 33 (35). 
diesem Zusammenhang auch vom europäischen „Staatenverbund“15, vom „Verfassungsverbund“ 16 und vom europäischen „Verwaltungs“- und „Verfassungsgerichtsverbund“17. Dementsprechend sind die einzelnen Mitgliedstaaten aufgefordert, völlig neue Instrumente, Regelungstypen und Konzepte mit weitreichenden Folgen in die eigene Rechtsordnung zu integrieren. ${ }^{18}$ Gleichzeitig greift mittlerweile auf europäischer Ebene eine eigenständige rechtliche und rechtswissenschaftliche Systembildung Platz, die z.B. berechtigt, vom „Europäischen Verwaltungsrecht“ zu sprechen, das „mehr ist als die Summe der durch das EU-Recht überformten und in diesem Sinne ‘europäisierten' nationalen Verwaltungsrechtsordnungen “. 19

\section{Internationalisierung und globaler Wettbewerb der Rechtssysteme}

Auch auf internationaler Ebene können wir seit geraumer Zeit Prozesse beobachten, die das nationale Rechtssystem nachhaltig beeinflussen: ${ }^{20}$ Die Ausdehnung wirtschaftlicher Austauschprozesse über nationale Grenzen hinweg, die Entwicklung weltumspannender Kommunikations- und Informationstechnologien, die Entstehung transnationaler Kapitalmärkte (und deren Zusammenbruch), den quantitativen Anstieg und Bedeutungszuwachs transnationaler Institutionen sowie global vernetzter Sozial- und politischer Bewegungen und transnationale Migration. ${ }^{21}$ Dieser Prozess der Internationalisierung - vielfach als „Globalisierung “ bezeichnet ${ }^{22}$ - und die

15 BVerfGE 123, 267 (348): „Der Begriff des Verbundes erfasst eine enge, auf Dauer angelegte Verbindung souverän bleibender Staaten, die auf vertraglicher Grundlage öffentliche Gewalt ausübt, deren Grundordnung jedoch allein der Verfügung der Mitgliedstaaten unterliegt und in der die Völker - das heißt die staatsangehörigen Bürger - der Mitgliedstaaten die Subjekte demokratischer Legitimation bleiben“; s. zum Begriff auch P. Kirchhof, Der europäische Staatenverbund, in: A. v. Bogdandy/J. Bast (Hrsg), Europäisches Verfassungsrecht, 2. Aufl., Berlin et al. 2009, 1009 (1019f.).

16 Vgl. Pernice, Die Dritte Gewalt im europäischen Verfassungsverbund, EuR 1996, 27 (33): „Der Begriff des Verfassungsverbunds kennzeichnet ... die materielle Einheit von Gemeinschafts- und innerstaatlichem (Verfassungs-)Recht; ders., Der Europäische Verfassungsverbund auf dem Wege der Konsolidierung, JöR 48 (1999), 205; ders., VVDStRL 60 (2001), 148 (172 f.); P.M. Huber, VVDStRL 60 (2001), 194 (199)).

17 Vgl. E. Schmidt-Assmann/B. Schöndorf-Haubold (Hrsg.), Der Europäische Verwaltungsverbund, Tübingen, 2005, sowie A. Voßkuble, Der europäische Verfassungsgerichtsverbund, NVwZ 2010, S. $1 \mathrm{ff}$.

18 A. Voßkuble, Methode und Pragmatik im Öffentlichen Recht, Methode und Pragmatik im Öffentlichen Recht, in: H. Bauer u.a. (Hrsg.), Umwelt, Wirtschaft, Recht, Tübingen 2002, S. 171 ff. (177).

19 E. Schmidt-Aßmann, Das allgemeine Verwaltungsrecht als Ordnungsidee, 2. Aufl., Berlin et al. 2004, S. 377.

20 R. Poscher, Das Verfassungsrecht vor den Herausforderungen der Globalisierung, VVDStRL 67, S. $160 \mathrm{ff}$., unterscheidet insoweit „faktische Globalisierungsphänomene“ einerseits und „regulative Globalisierungsphänomene" andererseits.

21 K. Günther/S. Randeria, Recht, Kultur und Gesellschaft im Prozess der Globalisierung, Bad Homburg 2001, S. 10.

22 Günther/Randeria, Recht (Fn. 21), S. 10. Vom „globalen Recht“ spricht z.B. G. Teubner, vgl. ders.: Societal Constitutionalism: Alternatives to State-Centred Constitutional Theory, in: C. Joerges/I.-JSand/G. Teubner (Hrsg.), Transnational Governance and Constituionalism, Oxford (Hart) S. $141 \mathrm{ff}$.; s. auch bereits ders., Global Law without a State, Farnham (Ashgate) 1997. Zum „Verfassungsrecht vor den Herausforderungen der Globalisierung” vgl. auch die Beiträge von R. Poscher und G. Nolte, VVDStRL 67, S. $160 \mathrm{ff}$. und $129 \mathrm{ff}$. sowie M. Ruffert, Die Globalisierung als Herausforderung an das Öffentliche Recht, Stuttgart et al. 2004. 
durch ihn produzierten Konflikte und Problemlagen müssen rechtlich verarbeitet werden.

Die damit in Zusammenhang stehenden rechtlichen Veränderungsprozesse lassen sich gut mit dem Stichwort der „Transnationalisierung des Rechts“ umschreiben. ${ }^{23}$ Dabei richtet sich der Blick nicht nur auf das wachsende internationale (zwischenstaatliche) Vertragsrecht, sondern auch auf halbstaatliche und nichtstaatliche Regelbestände wie transnationale (technische) Standardsetzung oder Standards internationaler Gremien (z.B. Luftverkehrssicherheit). ${ }^{24}$ Neben den nationalen Gesetzgebern operieren heute supranationale Gesetzgeber wie die Europäische Union und diverse internationale Organisationen wie die Welthandelsorganisation (WTO), die Weltbank oder der Internationale Währungsfonds (IWF). Daneben können wir halbstaatliche oder private Organisationen wie internationale Nichtregierungsorganisationen (NGOs) beobachten, die in der Grauzone zwischen der gesellschaftlichen Artikulation von Interessen und Ansprüchen und ihrer staatlichen Transformation in allgemein anerkannte Rechte verbindliche Regelungen schaffen. ${ }^{25}$

Das Recht und die rechtsrelevanten Normen als Gegenstände juristischer Tätigkeit entspringen nach alledem - jedenfalls faktisch - den unterschiedlichsten Rechtsquellen ${ }^{26}$ und werden erzeugt von Akteuren verschiedener Ebenen. ${ }^{27}$ In bestimmten Kon-

23 Vgl. Günther/Randeria, Recht (Fn. 21), S. 17: „Zunehmend wird ... das Recht in seiner grundlegenden Bedeutung nicht nur für die Transformation, sondern auch für die Erhaltung des Staates in einer globalisierten Welt wahrgenommen. Es erscheint uns daher sinnvoll, statt von 'Globalisierung' von 'Transnationalisierung' des Rechts zu sprechen, um so nicht nur auf den Prozesscharakter des Phänomens aufmerksam zu machen, sondern auch auf die nach wie vor wichtige, wenn auch sich nachhaltig verändernde Rolle nationaler Rechtssysteme und der Nationalstaaten. Während nationale Rechtsbereiche zunehmend transnationalisiert werden, durchdringen gleichzeitig internationale und supranationale Rechtsregime nationale Rechtsordnungen "; s. auch - grundlegend zur transnationalen Rechtserzeugung - H. H. Koh, The 1994 Roscoe Pound Lecture: Transnational Legal Process, in: Nebraska Law Review 75 (1996), S. 181 ff.; weitere Nachweise aus der US-amerikanischen Literatur bei F. Hanschmann, Theorie transnationaler Rechtsprozesse, in: S. Buckel/R. Christensen/A. FischerLescano (Hrsg.), Neue Theorien des Rechts, Stuttgart 2006, S. 34 ff.; vgl. ferner G. Brüggemeier (Hrsg.), Transnationalisierung des Rechts, Baden-Baden 2004. Zu neueren Entwicklungen der Theorie des globalen Rechts vgl. nur A. Fischer-Lescano/G. Teubner (Hrsg.), Regime-Kollisionen: Zur Fragmentierung des globalen Rechts, Frankfurt am Main 2006.

24 Vgl. T. Vesting, Rechtstheorie, München 2007, Rn. 147; zur Standardsetzung H.C. Röhl, Internationale Standardsetzung und O. Lepsius, Standardsetzung und Legitimation, jeweils in: C. Möllers/A. Voßkuhle/C. Walter (Hrsg.), Internationales Verwaltungsrecht, Tübingen 2007, S. $319 \mathrm{ff}$. und S. $345 \mathrm{ff}$.

25 Günther/Randeria: Recht (Fn. 21), S. 10; näher zu den einzelnen Akteuren der Transnationalisierung des Rechts ebd., S. $52 \mathrm{ff}$.

26 Der Ausdruck „Rechtsquelle” wird hier untechnisch verwendet - vgl. zur Problematik der herkömmlichen „Rechtsquellenlehre“ und der Frage einer Neukonzeption derselben M. Ruffert, Rechtsquellen und Rechtsschichten des Verwaltungsrechts, in: W. Hoffmann-Riem/E. Schmidt-Aßmann/A. Voßkuhle (Hrsg.), Grundlagen des Verwaltungsrechts, Bd. I, München 2006, $\$ 17$ Rn. 8 ff.; Vesting, Rechtstheorie (Fn. 24), Rn. $146 \mathrm{ff}$.

27 Vgl. R. Christensen/M. Böhme, Europas Auslegungsgrenzen: Das Zusammenspiel von Europarecht und nationalem Recht, Rechtstheorie 40 (2009), S. 285 ff. (289): „... Es geht also um die Vernetzung zweier autonomer Rechtsordnungen beim gemeinsam bestimmten Hervorbringen einer Entscheidung". 
stellationen (beispielsweise bei der richtlinienkonformen Auslegung nationaler Gesetze) kann es so zu einer Art rechtlicher „Koproduktion“ kommen, bei der zwei autonome Rechtsordnungen im gemeinsam bestimmten Hervorbringen einer Entscheidung vernetzt sind. ${ }^{28}$

Gleichwohl greift die Annahme, der Staat sei im Verlauf dieser Prozesse in eine rein passive Rolle gedrängt, zu kurz. ${ }^{29}$ Er trägt nicht nur die Gewährleistungsverantwortung. Vielmehr ist er aktiv dabei, neue Formen staatlichen Handelns und der Steuerung zu entwerfen, indirekte und direkte Steuerungsmechanismen zu kombinieren und neue legislative und exekutive Instrumente auszuprobieren. ${ }^{30}$ Neue Instrumente können durch internationale Harmonisierungsprozesse veranlasst sein oder zumindest von Vorbildern in anderen Ländern inspiriert werden. Sie können aber auch ihrerseits als erfolgreiche Modelle in den internationalen „Wettbewerb der rechtlichen Arrangements“ 31 eingespeist werden. Wie auch immer man dazu stehen mag, man wird festhalten können, dass die Grenzen nicht nur für Informationen, Ideen, Kapital- und Warenströme durchlässiger geworden sind, sondern auch für Rechtsformen, juristische Argumentationsmuster und Verfahrensmodalitäten. ${ }^{32}$

Auch die Judikative bleibt von dem internationalen Wettbewerb der rechtlichen Konzepte, Ideen und Arrangements nicht unbeeinflusst. Veränderungsprozesse der Justiz, die nicht zuletzt durch erfolgreiche Modelle anderer Länder beeinflusst sind, manifestieren sich beispielsweise in der zunehmenden Etablierung alternativer Kon-

28 Christensen Böhme, Auslegungsgrenzen (Fn. 27), S. 289.

29 Vgl. zum Wesen des Engagements des Staates im Prozess der Globalisierung S. Sassen, Paradox des Nationalen: Territorium, Autorität und Rechte im globalen Zeitalter, Frankfurt am Main 2008, insbes. S. $371 \mathrm{ff}$.

30 Sassen, Paradox (Fn. 29), S. 371.

31 Vgl. zu diesem Wettbewerb - mit deutlich kritischer Note - Sassen, Paradox (Fn. 29), S. 371 f.: „Einige Staaten ... produzieren die Entwürfe für diese neuen 'Rechtlichkeiten' und nötigen diese aufgrund der Interdependenzen, die für das gegenwärtige Stadium der Globalisierung zentral sind, den anderen Staaten auf. Doch auch dann müssen die beteiligten Staaten die spezifischen Instrumente im Hinblick auf ihre jeweiligen politisch-ökonomischen Arrangements entwickeln.“ Ebd., Fn. 6: „... Frankreich z.B. zählt zu den führenden Anbietern von Dienstleistungen im Bereich der Information und des industriellen Ingenieurswesens und nimmt bei Finanz- und Versicherungsdienstleistungen eine starke, wenn auch nicht herausragende Position ein. Doch sieht sich Frankreich bei rechtlichen Dienstleistungen und solchen im Rechnungswesen zunehmend benachteiligt, da anglo-amerikanisches Recht und anglo-amerikanische Standards bei internationalen Geschäftsabschlüssen dominieren. Angloamerikanische Firmen mit Niederlassungen in Paris arbeiten den juristischen Bedürfnissen der Formen, die von Frankreich aus operieren, zu - ganz gleich, ob dies französische oder ausländische Formen sind. Ähnlich dominiert das anglo-amerikanische Recht immer stärker das internationale Handelsschiedswesen, dessen institutionelle Grundlagen auf kontinentalen - vor allem französischen und schweizerischen - Traditionen der Rechtsprechung aufbauen."

32 R. Voigt, Globalisierung des Rechts. Entsteht eine „dritte Rechtsordnung“?, in: ders. (Hrsg.), Globalisierung des Rechts, Baden-Baden 2000, S. 13 ff. (19). 
fliktlösungsmechanismen wie der Mediation, ${ }^{33}$ aber auch in der Betonung judikativer Transparenz, der nachvollziehbaren, guten Begründung von Entscheidungen und der Bürgerfreundlichkeit der Justiz. Die Justiz wird insoweit zunehmend auch als ein Standortfaktor wahrgenommen.

Als weiterer Beitrag im „Wettbewerb der rechtlichen Arrangements“ dürfte auch die Initiative der Länder Nordrhein-Westfalen und Hamburg anzusehen sein, Kammern für internationale Handelssachen (KfiHG) einzuführen, vor denen auf Englisch verhandelt werden können soll. Hier wird ausdrücklich argumentiert mit der - ich zitiere - „Bedeutung des globalen Standortwettbewerbs, der auch die Justiz erfasst hat“34. Wir können darin den Versuch sehen, globalen Unternehmen ein „klassisches“ Konfliktlösungsmodell - nämlich den Prozess vor einem staatlichen Gericht - als Alternative zu internationalen Handelsschiedsverfahren schmackhaft zu machen, die derzeit in der Regel durch private Schiedspersonen entschieden werden. ${ }^{35}$ Ohne an dieser Stelle diese Initiative zur Einführung von englischsprachigen Kammern für internationale Handelssachen rechtlich oder rechtspolitisch abschließend bewerten zu wollen: Sie ist jedenfalls ein Beleg dafür, dass die Wahrnehmung eines globalen Wettbewerbs der Rechtssysteme ${ }^{36}$ vor der Judikative nicht halt macht. ${ }^{37}$

\section{Wissensgesellschaft: Wissen, Nichtwissen, Digitalisierung}

Internationalisierung und Globalisierung werden ihrerseits begleitet von einer weiteren Entwicklung, die gemeinhin unter dem schillernden Schlagwort der „Wissens-

33 In diese Richtung auch I. v. Münch, Legal Education and the Legal Profession in Germany, BadenBaden 2002, S. 71: „Perhaps a development towards more mediation instead of court decisions will take place“; D. Strempel, Neuer Ausbildungsinhalt: Außergerichtliche Konfliktregelung (Mediation), in: ders. (Hrsg.), Juristenausbildung zwischen Internationalität und Individualität - auch ein Problem der Gesetzgebung, Baden-Baden 1998, S. 365 ff.; zur Mediation vgl. aus neuerer Zeit J. v. Bargen, Mediation im Verwaltungsprozess, DVBl. 2004, S. 468 ff.; ders., Gerichtsinterne Mediation, Tübingen 2008; R. Pitschas, Mediation als Methode und Instrument der Konfliktmittlung im öffentlichen Sektor, NVwZ 2004, S. 396 ff.; U. Rüssel, Mediation in komplexen Verwaltungsverfahren, Baden-Baden 2004.

34 Pressemitteilung des Justizministeriums Nordrhein-Westfalen vom 12. Februar 2010 - „Englisch als mögliche Gerichtssprache: Justizministerin Roswitha Müller-Piepenkötter stellt Gesetzentwurf im Bundesrat vor“ (abrufbar unter http://www.justiz.nrw.de/Presse/PresseJM/12_02_10/index.php, Zugriff vom 25.3.2010).

35 Vgl. BR-Drucks. 42/10 vom 27.01.10, S. 2: „Der Gerichtsstandort Deutschland wird durch die Einführung von Englisch als Gerichtssprache in hohem Maße an Attraktivität gewinnen. Deutsche Kammern für internationale Handelssachen werden bedeutende wirtschaftsrechtliche Verfahren anziehen, die bisher entweder vor Schiedsgerichten oder im englischsprachigen Ausland verhandelt werden. Die zunehmende Vereinbarung des Gerichtsstandortes Deutschland wird auch die vermehrte Wahl des deutschen Rechts als auf internationale Vertragsverhältnisse anwendbares Recht nach sich ziehen“.

36 Zum „Wettbewerb der Rechtsordnungen” vgl. die Referate von A. Peters und T. Giegerich auf der Staatsrechtslehrertagung 2009 in Graz, VVDStRL 69 (2010), S. 7 ff. bzw. 57 ff. L. Michael, Wettbewerb von Rechtsordnungen, DVBl. 2009, S. $1062 \mathrm{ff}$.

37 Skeptischer dagegen von Münch, Legal Education (Fn. 33), S. 71: „With regard to the future of legal professions, the profession of a judge will probably not undergo remarkable changes. Perhaps a development towards more mediation instead of court decisions will take place. Efficient administrative steps, with the use of new technologies, will have to be implemented to alleviate the burden of the courts." 
gesellschaft" diskutiert wird ${ }^{38}$ : Bis 2010 wollte die EU nach einer Erklärung des Europäischen Rates im Jahr 2000 zur „wettbewerbsfähigsten Wissensgesellschaft der Welt“ geworden sein. ${ }^{39}$ Auch in der „Bologna-Erklärung“ aus dem Jahr 1999 ist die Rede von dem „Europa des Wissens“. Es heißt dort: „Inzwischen ist ein Europa des Wissens weitgehend anerkannt als unerlässliche Voraussetzung für gesellschaftliche und menschliche Entwicklung sowie als unverzichtbare Komponente der Festigung und Bereicherung der europäischen Bürgerschaft ... “40

Andererseits wächst unser Problembewusstsein für Ungewissheiten: Die Generierung neuen wissenschaftlichen Wissens lässt zugleich auch das scheinbar „unbegrenzte Universum des Nichtwissens “ (Weingart) deutlicher sichtbar werden. ${ }^{41} \mathrm{Im}$ Zentrum der Wissensgesellschaft steht aus diesem Blickwinkel die Generierung von Expertise im Umgang mit Nichtwissen. ${ }^{42}$ Im Verwaltungsrecht wird diese Entwicklung besonders augenfällig, wenn es beispielsweise um das Planungs-, Umwelt- und Technikrecht geht. Hier erweist sich die Entscheidungssituation aufgrund der zu bewältigenden Zielkonflikte, der Unsicherheit der Zukunft und den in aller Regel bestehenden Wahlmöglichkeiten zwischen verschiedenen Handlungsalternativen häufig als außerordentlich komplex. ${ }^{43}$

Das vorhandene Wissen ist überdies durch seine zunehmende Digitalisierung neu geordnet, anders verknüpft und schier unbegrenzt verfügbar. Auch das rechtliche Wissen ist digitalisiert und wird erst durch die jeweilige Technik des Zugriffs geordnet, möglicherweise also - je nach individuellem Bedarf - unsystematisch, nichthier-

38 Zum Verhältnis von Wissen und Recht vgl. hier nur statt vieler die Beiträge in I. Spiecker gen. Döhmann/P. Collin (Hrsg.), Generierung und Transfer staatlichen Wissens im System des Verwaltungsrechts, Tübingen 2008; G. F. Schuppert/A. Voßkuble (Hrsg.), Governance von und durch Wissen, Baden-Baden 2008, sowie H. C. Röhl (Hrsg.), Wissen - Zur kognitiven Dimension des Rechts, Beiheft 9 zu Die Verwaltung, 2010. Allgemein zur Problematik auch B. Faßbender, Wissen als Grundlage staatlichen Handelns, in: J. Isensee/P. Kirchhof (Hrsg.), Handbuch des Staatsrechts der Bundesrepublik Deutschland, Bd. IV, 3. Aufl., Heidelberg 2006, $\mathbb{} 76$.

39 Vgl. A. v. Bogdandy, Entgrenzung der Wissenschaften und Prämissen des europäischen Wissenschaftsraums: Anregungen zur Gestaltung des Europäischen Forschungsrats, in: HRK (Hrsg.), Beiträge zur Hochschulpolitik 2/2007, 69 (70) mit Verweis auf die Schlussfolgerungen des Vorsitzes, Europäischer Rat (Lissabon), 23. und 24. März 2000 (SN 100/00), Nr. 5: Nach dem ersten Gipfel solle die Europäische Union bis zum Jahr 2010 zur wettbewerbsfähigsten Wissensgesellschaft der Welt werden.

40 Der Europäische Hochschulraum. Gemeinsame Erklärung der Europäischen Bildungsminister, 19. Juni 1999, Bologna, abrufbar unter www.bmbf.de/pub/bologna_deu.pdf (Zugriff vom 1.4.2010): „Inzwischen ist ein Europa des Wissens weitgehend anerkannt als unerläßliche Voraussetzung für gesellschaftliche und menschliche Entwicklung sowie als unverzichtbare Komponente der Festigung und Bereicherung der europäischen Bürgerschaft ..."

41 A. Voßkuble, Expertise und Verwaltung, in: H.-H. Trute/T. Groß/H. C. Röhl/C. Möllers (Hrsg.), Allgemeines Verwaltungsrecht - zur Tragfähigkeit eines Konzepts, Tübingen 2008, S. 637 ff. (652) m.w.N.; A. Scherzberg. Wissen, Nichtwissen und Ungewissheit im Recht, in: C. Engel/J. Halfmann/ M. Schulte (Hrsg.), Wissen - Nichtwissen - Unsicheres Wissen, Baden-Baden 2002, S. $113 \mathrm{ff} . ;$ I. Apel, Methodik des Umgangs mit Ungewissheit, in: Schmidt-Aßmann/Hoffmann-Riem (Hrsg.), Methoden (Fn. 8), S. 327 ff. sowie die Beiträge in: I. Augsberg (Hrsg.), Unwissen als Chance, Tübingen 2009.

42 Voßkuble, Expertise (Fn. 41), S. 653.

43 Voßkuble, Expertise (Fn. 41), S. 643. 
archisch: Hier entwickelt sich in der Praxis ein ganz neues methodisches oder bisweilen auch nichtmethodisches Vorgehen, dessen Einfluss auf die Rechtserzeugung noch gar nicht abschätzbar ist. ${ }^{44}$ Die Veränderungsprozesse, die wir unter dem Schlagwort „Digitalisierung des Staates“ zusammenfassen können, greifen aber noch weiter - man denke an Projekte wie die „elektronische Akte“, den teilweise bereits praktizierten „elektronischen Rechtsverkehr“ und das „e-Government“. 45

\section{Folgerungen für die juristische Ausbildung in Deutschland: Das Leitbild des europäischen Juristen}

Was folgt aus den hier nur in aller Kürze skizzierten Veränderungsprozessen für das Leitbild des europäischen Juristen und die daran orientierte Ausbildung?

\section{Rechtsanwendung und Rechtserzeugung - der europäische Jurist als Akteur in nationalen, europäischen und internationalen Rechtserzeugungsprozessen}

Mehr als 130 Jahre nach Vereinheitlichung der Juristenausbildung durch die Reichsjustizgesetze (1877) ${ }^{46}$ soll sich der in Deutschland ausgebildete europäische Jurist in internationalen Diskussionszusammenhängen zurecht finden können, mehr noch, er soll gemeinsame Themen mit europäischen Juristen aus anderen Ländern finden, er soll mit Juristen aus anderen Ländern eine im übertragenen Sinne „gemeinsame Sprache“ entwickeln, eigene Errungenschaften in den internationalen Diskussions-

44 Vgl. schon F. Haft, Das Internet in der Juristenausbildung, in: D. Strempel (Hrsg.), Juristenausbildung (Fn. 33), S. 123 ff. (124): „Juristisches Wissen ist in geschriebenen und gedruckten Texten gespeichert, die schon längst zu einer gewaltigen Informationslawine angeschlossen sind. Bereits in den sechziger Jahren hat man die Möglichkeit des Computereinsatzes zur Bewältigung dieser Lawine erkannt und seitdem große juristische Datenbanken wie juris und Lexinform geschaffen ... Die verwendeten Suchtechniken über Begriffe und Begriffsverknüpfungen sind freilich durch und durch unjuristisch “; H.-J. Strauch, Wandel des Rechts durch juristische Datenbanken?, DVBl. 2007, S. $1000 \mathrm{ff}$.

45 S. dazu den „Aktionsplan Deutschland-Online“, Beschluss der Regierungschefs des Bundes und der Länder vom 22.6.2006, neu gefasst am 19.11.2009 (abrufbar unter www.deutschland-online.de, Zugriff vom 1.4.2010). Dazu zuletzt R. F. Heller / E. Richter, Das Recht als erfolgskritischer und reformstrategischer Faktor im E-Government - Dargestellt anhand der praktischen Erfahrungen bei der Umsetzung des prioritären Vorhabens „KfZ-Wesen“ des Aktionsplans Deutschland-Online, DVBl. 2010, S. 345 ff. (346). Allgemein zum E-Government M. Eifert, Electronic Government, Baden-Baden 2006; G. Britz, Elektronische Verwaltung, in: Hoffmann-Riem/Schmidt-Aßmann/Voßkuhle (Hrsg.), Grundlagen (Fn. 26), Bd. II, München 2008, \$26; H. Hill/ U. Schliesky (Hrsg.), Herausforderung eGovernment, Baden-Baden 2009; V. Böhme-Neßler: Das Ende des Staates? Zu den Auswirkungen der Digitalisierung auf den Staat, ZÖR 64 (2009), S. 145 ff.; ders., Unscharfes Recht: Überlegungen zur Relativierung des Rechts in der digitalisierten Welt, Berlin 2008.

46 Pieroth, Literarische Streifzüge (Fn. 9), S. 795. 
zusammenhang einspeisen ${ }^{47}$ und in Aushandlungsprozessen bestehen und in deren Rahmen Konflikte lösen. ${ }^{48}$

Der Jurist hat sich damit vom gesetzespositivistischen „Diener des Nationalstaates“ - wie er von Hermann Kantorowicz überzeichnet dargestellt wurde ${ }^{49}$ - zum Begleiter und Akteur vielfältiger Rechtsentstehungsprozesse gewandelt, die auf den unterschiedlichsten, miteinander verschränkten inner-, intra- oder interstaatlichen, halbstaatlichen oder nichtstaatlichen Ebenen stattfinden.

Leitbild der Juristenausbildung wird danach nicht mehr der das nationale Recht subsumierende Richter sein. Aber auch der globale „Wall Street Lawyer“ nach amerikanischem Muster - der für die große Mehrzahl der in Deutschland tätigen Rechtsanwälte ohnehin nicht paradigmatisch gewesen sein dürfte - scheidet nicht erst seit der Finanzkrise als Leitbild des europäischen Juristen aus. ${ }^{50}$ Paradigmatisch und zukunftsweisend ist vielmehr der vielfältig einsetzbare Rechtsgestalter, der über Orientierungs- und Verfügungswissen für soziales Handeln in einer komplexen Welt verfügt und der bei zunehmender europäischer und internationaler Verflechtung der Rechtssysteme im „Wettbewerb rechtlicher Arrangements“ selbst Vorschläge zu formulieren und in die Normerzeugungsprozesse auf europäischer und internationaler Ebene bereits im Vorfeld einzuspeisen vermag. ${ }^{51}$

Die juristische Ausbildung muss dieser Vielfalt juristischer Tätigkeit Rechnung tragen: Die Jurisprudenz ist nicht nur Rechtsanwendungs-, sondern auch Rechtsetzungs-, Rechtsgestaltungs-, Entscheidungs- und Handlungswissenschaft; der Jurist „als solcher" ist nicht mehr nur der Richter, sondern er ist auch Rechtsberater, Rechtsgestalter, Rechtserzeuger. An die Stelle einer national introvertierten Norm-

47 C. Möllers/A. Voßkuble, Die deutsche Staatsrechtswissenschaft im Zusammenhang der internationalisierten Wissenschaften, Die Verwaltung 36 (2003), 321 (331).

48 Vgl. z.B. zu Aushandlungsprozessen im Komitologie-Verfahren: J. Falke, in: C. Joerges/J. Falke (Hrsg.), Das Ausschusswesen in der Europäischen Union, Baden-Baden 2000, S. 43 ff.; zur Mediation - auch an den Gerichten - vgl. bereits oben bei Fn. 33; allgemein zur Konfliktlösung durch Verhandlung W. Hoffmann-Riem, Konfliktmittler in Verwaltungsverhandlungen, Heidelberg 1989; B. Holznagel, Konfliktlösung durch Verhandlungen, Hamburg 1990; W. Hoffmann-Riem/E. Schmidt-Aßmann (Hrsg.), Konfliktbewältigung durch Verhandlungen, Bd. I und II, Baden-Baden 1990.

49 Vgl. für eine differenzierte Sicht auf die Praxis des Reichsgerichts J. Schröder, Zum Gesetzespositivismus in der Rechtsprechung des Reichsgerichts, in: G. Kohl/C. Neschwara/T. Simon (Hrsg.), FS für W. Brauneder zum 65. Geburtstag, Wien 2008, S. 603 ff.

50 Vgl. zum Niedergang des „Wall Street Lawyers” aus der US-amerikanischen Literatur: L. Ribstein, The Death of Big Law, abrufbar unter www.law.georgetown.edu/ LegalProfession/documents/ Ribstein.pdf (Zugriff vom 1.4.2010); T. D. Morgan, The Vanishing American Lawyer, New York (Oxford University Press) 2010 und ders., The Last Days of the American Lawyer, abrufbar unter http://papers.ssrn.com/abstract=1543301 (Zugriff vom 1.4.2010); M. C. Reagan Jr., Eat What You Kill: The Fall of a Wall Street Lawyer, Ann Arbor (University of Michigan Press) 2005; R. Susskind, The End of Lawyers? Rethinking the Nature of Legal Services, Oxford (Oxford University Press) 2008.

51 Vgl. schon Voßkuble, Methode (Fn. 18), S. 178. 
und Rechtsprechungsexegese tritt ein problemorientierter Austausch von rechtlichen Argumenten, Lösungsansätzen und Erfahrungen. ${ }^{52}$

Aus dieser Erweiterung des Tätigkeitsfeldes folgt zugleich, dass das Leitbild der juristischen Ausbildung der europäische Jurist als umfassend ausgebildeter Einheitsjurist sein muss: Gerade aufgrund der Vielzahl denkbarer Perspektiven und Rollen muss die Ausbildung gewährleisten, dass jede von ihnen potentiell eingenommen werden kann.

\section{Internationalität - der europäische Jurist als juristischer Kosmopolit}

So, wie der europäische Jurist sich auf unterschiedliche Perspektiven juristischen Denkens und Handelns einlassen können muss, muss er auch in der Lage sein, die diversen Ebenen des Rechts stets „mitzudenken“ und sich auf andere juristische Traditionen und Denkstile einzulassen. Damit bin ich bei meinem nächsten Punkt angelangt, dem europäischen Juristen als „juristischem Kosmopolit“.

Bei der europäischen und internationalen Dimension der juristischen Ausbildung, die ich meine, geht es weniger um die formale, strukturelle Harmonisierung des Studienablaufs. Denn mit einer bloß formalen Vereinheitlichung ist noch nichts gewonnen, sondern möglicherweise sogar nur etwas verloren. Es geht vielmehr darum, das juristische Denken und Handeln und damit auch die juristische Ausbildung in Deutschland inhaltlich auf Europa und die Welt auszurichten. ${ }^{53}$ Gefordert ist in den Worten von Peter Häberle (der als erster vom „europäischen Juristen“ gesprochen hat ${ }^{54}$ ) dass

„jeder Jurist im Alltag national und europäisch arbeitet, d.h.: z.B. als nationaler Richter im Rahmen des Europarates sich auch als EMRK-Richter versteht bzw. im Rahmen der EU auch als 'EU-Gemeinschaftsrichter' entscheidet: und zwar in allen Instanzen und auf allen Ebenen der nationalen Rechtsordnungen. Entsprechendes gilt für die Aufgaben des Rechtsanwaltes, des Rechtsberaters und vor allem der Professoren und Studenten an juristischen Fakultäten... "55

52 Voßkuble, Methode (Fn. 18), S. 178 f.; ders., Die Renaissance der „Allgemeinen Staatslehre“ im Zeitalter der Europäisierung und Internationalisierung, JuS 2004, S. 1 ff. (5) m.w.N.

53 Vgl. Böttcher, Jurist (Fn. 4), S. 9: „Die vergleichende Betrachtung der Strukturen der Juristenausbildung in Europa ist eine Sache, wenn man vom europäischen Juristen spricht. Die inhaltliche Ausrichtung der Juristenausbildung auf Europa ist eine andere“.

54 Vgl. die Nachweise in Fn. 8; zur Würdigung Pernice, Peter Häberle (Fn. 8), S. 9: „Er ist der Europäische Jurist, einer, der diese Bezeichnung verdient, wie kaum ein anderer."

55 P. Häberle, Der europäische Jurist (Fn. 8); vgl. auch ders., Der europäische Jurist, JöR 50 (2002), S. $123 \mathrm{ff}$. 
Hinzuzufügen wäre die internationale Dimension. In Zeiten, in denen von einer „Konstitutionalisierung des Völkerrechts“56 und einer „globalisierten Jurisprudenz" 57 ebenso wie von einer „Fragmentierung“58 und einem „Pluralismus“59 des (globalen) Rechts gesprochen werden kann, ist Leitbild des europäischen Juristen auch der „juristische Kosmopolit“ - der juristische Kosmopolit mit Bewusstsein für die außereuropäischen Rechtsordnungen, mit Bewusstsein für deren Konvergenzen sowie für deren gewachsene Eigenständigkeit und Eigenart sowie einem Gespür für globale Normentwicklungen. ${ }^{60}$ Auch in Bezug auf die internationalen Normentstehungsprozesse gilt es deshalb, ein Gefühl der „Mit-Verantwortlichkeit“ zu entwickeln, so, wie es Hans Peter Ipsen bereits im Jahr 1964 auf dem Karlsruher Juristentag für das europäische Gemeinschaftsrecht gefordert hatte. ${ }^{61}$

Voraussetzung für all dies sind Rechtskenntnisse im Europarecht, im internationalen und ausländischen Recht sowie Methodenkompetenz im Bereich der Rechtsvergleichung - für die es mehr bedarf als eines bloßen Textvergleichs, denn die jeweiligen

56 Vgl. J. Habermas, Konstitutionalisierung des Völkerrechts und die Legitimationsprobleme einer verfassten Weltgesellschaft, in: W. Brugger/U. Neumann/S. Kirste (Hrsg.), Rechtsphilosophie im 21. Jahrhundert, Frankfurt am Main 2008, S. 360 ff.; A. L. Paulus, Zur Zukunft der Völkerrechtswissenschaft in Deutschland: zwischen Konstitutionalisierung und Fragmentierung des Völkerrechts, ZaöRV 67 (2007), S. 695 ff.; J. A. Frowein, Konstitutionalisierung des Völkerrechts, in: ders., Völkerrecht Menschenrechte - Verfassungsfragen Deutschlands und Europas, Berlin et al. 2004, S. 173 ff.; B.-O. Bryde, Konstitutionalisierung des Völkerrechts und Internationalisierung des Verfassungsrechts, Der Staat 42 (2003), S. 61 ff.; allgemein G. F. Schuppert/C. Bumke, Die Konstitutionalisierung der Rechtsordnung, Baden-Baden 2000.

57 C. Möllers, Globalisierte Jurisprudenz, ARSP-Beiheft 79 (2001), S. 41 ff.

58 Vgl. nur Fischer-Lescano/Teubner, Regime-Kollisionen (Fn. 23).

59 Vgl. zur Theorie des „legal pluralism” den Überblick bei R. Cotterrell, Transnational Communities and the Concept of Law, Ratio Juris vol. 21 March 2008, p. $1 \mathrm{ff}$. (8ff.) und (7): „... In fact, almost all theoretically sophisticated socio-legal writing on globalisation and law adopts a legal pluralist perspective. What is such a perspective? The term 'legal pluralism' normally refers to 'a situation in which two or more legal systems coexist in the same field' (Merry, 1988, 870), making independent jurisdictional claims".

60 Vgl. zum globalisierten geisteswissenschaftlichen Diskurs Möllers/Voßkuble, Staatsrechtswissenschaft (Fn. 47), S. 330 f.; C. Möllers, Globalisierte Jurisprudenz, ARSP-Beiheft 79 (2001), S. 41 ff.; mit Mahnung zur Selbstkritik und Bescheidenheit P. Häberle, Der europäische Jurist, (Fn. 8), S. 32 f.: „Die vermeintliche oder wirkliche „Universalität“ mancher europäischer Rechtsprinzipien darf nicht zum Instrument der Einebnung der Kulturen anderer Völker werden ... was nicht ausschließt, im Sinne I. Kants in „weltbürgerlicher Absicht“ zu denken und zu handeln ... Aber die Europäisierungsvorgänge sollen taktvoll und sensibel auf Europa beschränkt bleiben und allenfalls als „Angebot“ unterbreitet werden, nicht als verdeckte Form neuer Kolonialisierung."

61 H. P. Ipsen, Der deutsche Jurist und das europäische Gemeinschaftsrecht, Verhandlungen des fünfundvierzigsten deutschen Juristentages, Karlsruhe 1964, Bd. II, 1965, S. L 5 ff. (L 7): „Anders als gegenüber dem nationalen deutschen Binnenrecht, in dessen Behausung der deutsche Jurist sich aus Erziehung, Erkenntnis und Erfahrung heimisch fühlt, geht ihm gegenüber dem Gemeinschaftsrecht das Gefühl der Mit-Verantwortlichkeit ab. Er sieht diese Rechtsmasse werden und wachsen ohne Fähigkeit und Vermögen, an dem neuen Prozess der Rechtsgestaltung in etwa derart verantwortlich mitzuwirken, wie ihm das - als pragmatischem Juristen aller denkbaren beruflichen Standorte, nicht weniger als intellektuell und professionell dazu besonders berufenem Staatsbürger - in der Fortentwicklung der binnendeutschen Rechtsordnung möglich ist." 
Regelungen müssen historisch und kulturell kontextualisiert werden. ${ }^{62}$ Dabei kann es im Rahmen der Ausbildung nicht um verästelte Detailkenntnisse gehen, sondern lediglich darum, dem jungen Juristen einen verlässlichen Zugang zu eröffnen und seine „Mit-Verantwortlichkeit“ zu entwickeln.

Als „juristischer Kosmopolit“ benötigt der europäische Jurist Sprachkompetenz vor allem sollte er Englisch beherrschen und daneben möglichst noch eine weitere wichtige Fremdsprache. ${ }^{63}$ Man denke nur an die Urteile des zunehmend bedeutsamen Europäischen Gerichtshofs für Menschenrechte (EGMR): Sie werden amtlich nur auf Französisch und Englisch verfasst. Deutsche Übersetzungen sind manchmal mit Glück erhältlich, wenn sich eine Fachzeitschrift zur Veröffentlichung der Entscheidung in deutscher Übersetzung entschließt. Trotzdem müssen die Entscheidungen des EGMR bei der Auslegung des nationalen Rechts berücksichtigt werden. ${ }^{64}$ In Zukunft werden wir daher bei der juristischen Ausbildung dem Erwerb juristischer Fremdsprachenkenntnisse auch jenseits von Passau und Trier, die hier vorbildlich sind, noch mehr Raum einräumen müssen.

Das Stichwort „Raum einräumen“ führt mich hin zu einem weiteren Punkt, nämlich der Frage der Mobilität. Sprachkompetenz und Verständnis für andere Rechtssysteme oder wenigstens Begeisterung und die Bereitschaft, sich diese zu erschließen, erfährt man wahrscheinlich am schönsten im unmittelbaren Erlebnis eines Auslandsaufenthaltes (auch wenn nicht jeder Studienaufenthalt in Bologna uns die spontane Kreativität eines Hermann Kantorowicz verleihen mag). Das politische Plädoyer für eine weitere Verkürzung der Studiendauer halte ich auch aus diesem Grunde für verfehlt. Wer während des Studiums ins Ausland gehen soll, benötigt nämlich vor allem eins: Zeit.

62 Zur Rechtsvergleichung im Privatrecht vgl. die Werke von E. Rabel, M. Rheinstein, K. Zweigert; zur Rechtsvergleichung im Öffentlichen Recht vgl. nur P. Häberle, Rechtsvergleichung im Kraftfeld des Verfassungsstaates, Berlin 1992; G. Trantas, Die Anwendung der Rechtsvergleichung bei der Untersuchung des öffentlichen Rechts, Dresden 1998; R. Wahl, Verfassungsvergleichung als Kulturvergleichung, in: ders., Verfassungsstaat, Europäisierung, Internationalisierung, Frankfurt am Main 2003, S. $96 \mathrm{ff}$.

63 Vgl. A. v. Bogdandy, Beobachtungen zur Wissenschaft vom Europarecht, Der Staat 40 (2001), S. 3 ff. (42): „... Die Sprache dieser Metastruktur wird das Englische sein, und die englischen Rechtsbegriffe werden englische Rechtsbegriffe transportieren ... Die Übertragung kontinentaler öffentlich-rechtlicher Konstrukte ... wird der Selbsttransparenz dienen, den Gedanken schärfen, vielleicht sogar - langfristig - auf die tendenziell hermetischen nationalen Argumentationskulturen modifizierend zurück wirken“; Böttcher, Jurist (Fn. 4), S. 9: „Zur inhaltlichen Ausrichtung der Juristenausbildung auf Europa gehört die Integration von Sprachunterricht (...) Es kann nicht zweifelhaft sein, daß der europäische Jurist in Zukunft englisch in Wort und Schrift beherrschen sollte, möglichst noch eine weitere europäische Sprache“; Günther/Randeria, Recht (Fn. 21), S. 106: „Von besonderer Bedeutung sind unterschiedliche Arten von „Fernkompetenz“, also vor allem linguistische Kompetenzen, eine Sensibilität für die kulturellen Unterschiede (und zwar als intellektuelles Vermögen und nicht als Kurztraining in interkultureller Kommunikation), und die Fähigkeit zur Wahrnehmung differenter sozialer wie historischer Kontexte".

64 Vgl. BVerfGE 111, $307 \mathrm{ff}$. 


\section{Juristische Allgemeinbildung und Überblickswissen - der europäische Jurist als Wissensexperte mit rechtlichem Meta-Wissen}

Damit komme ich zu meiner dritten Schlussfolgerung: der europäische Jurist ist als Generalist „Wissensexperte“ mit rechtlichem „Meta-Wissen“65.

Soll der europäische Jurist flexibel mit Veränderungen umgehen können und sich in den konvergierenden Rechtsordnungen - die gleichwohl plural und unterschiedlich bleiben werden - zurechtfinden, kann es schon aus praktischen Erwägungen nicht darum gehen, als Ziel der Ausbildung noch breitere, umfassendere Kenntnisse vom positiven Recht und seinen weitreichenden Verästelungen zu verlangen. Allein das Europarecht ist heutzutage ein Rechtsgebiet, das in zahllose eigenständige Rechtsgebiete untergliedert ist, die ihrerseits eine enorme Komplexität und Ausdifferenzierung erreicht haben. Will man die Ausbildungszeit nicht ins Unendliche verlängern, scheiden die pauschale Erweiterung des Stoffs als Weg der Ausbildung und der „positivistische Stoffhuber" als Leitbild aus. Es kann also nur darum gehen, Methoden zu beherrschen, anhand derer das verfügbare Wissen im Bedarfsfalle abgerufen und erschlossen werden kann.

Das bedeutet auch, dass der „Spezialist“ als Leitbild ausscheidet, zumal innerhalb weniger Jahre ganze Rechtsgebiete neu entstehen können (man denke beispielsweise an „IT-Recht“), während andere womöglich in die Bedeutungslosigkeit hinabsinken. ${ }^{66}$ Trotzdem widerspricht es der Orientierung am Leitbild des Generalisten und „Wissensexperten“ nicht, eine gewisse Schwerpunktsetzung in Studium und Referendariat zu ermöglichen. Eine zu frühe oder zu starke Spezialisierung wird aber stets zu Lasten der Flexibilität des jungen Juristen gehen, und eben diese Flexibilität wird er angesichts der fortschreitenden Geschwindigkeit der Veränderungen im Rechtssystem dringend benötigen.

\section{Wissenschaftlichkeit und Grundlagenorientierung - der europäische Jurist als theoretisch und wissenschaftlich fundierter Praktiker}

Aus den gleichen Erwägungen heraus sollten Theorie und Praxis der juristischen Ausbildung weiterhin in einem zweistufigen, „dualen“ System voneinander grundsätzlich getrennt gehalten werden. ${ }^{67} \mathrm{~W}$ ir brauchen das wissenschaftliche Selbstver-

65 Voßkuble, Expertise (Fn. 41), S. 657 f.

66 Vgl. bereits Stolleis, Leitbild (Fn. 8), S. 200, der von einer „Verschiebung der Schwerpunkte im Inneren der Fakultäten, vornehmlich unter Einfluss des Marktes“"spricht.

67 Zu früheren Versuchen mit der „einstufigen Juristenausbildung “vgl. nur die Beiträge in W. Hassemer/ W. Hoffmann-Riem/J. Limbach (Hrsg.), Juristenbildung zwischen Experiment und Tradition, BadenBaden 1986. 
ständnis ${ }^{68}$ des europäischen Juristen ebenso wie die Wissenschaftlichkeit seiner Ausbildung. Die universitäre Juristenausbildung darf - mit anderen Worten - keine reine Berufsqualifikation sein, sondern sie muss dem „Freiheitsgedanken des wissenschaftlichen Erkenntnisprozesses “69 verbunden bleiben. Die Devise bleibt: „erst die Theorie, dann die Praxis“ “. ${ }^{70}$ Denn nirgendwo wird Praxis besser gelernt als eben in der Praxis. ${ }^{71}$ Das nötige Fundament, das aus einem schlichten Rechtsanwender einen guten Juristen macht, kann dagegen nur eine grundlagenorientierte, wissenschaftliche Ausbildung an der Universität vermitteln. Nicht von ungefähr vermeiden gerade die besseren Law Schools in den USA eine Annäherung an die Praxis, während die schlechteren hier ihre Chancen wittern. ${ }^{72}$

Damit soll nicht gesagt sein, die wissenschaftliche, grundlagenorientierte Ausbildung an der Universität hätte ihrerseits keinen praktischen Sinnbezug. Im Gegenteil: Die Rechtswissenschaft selbst war immer eine auf die Praxis bezogene Wissenschaft: Sie zielt nicht nur auf ein methodisch erarbeitetes, systematisch geordnetes, objektiv gesichertes, lehr- und lernbares rationales Wissen vom Recht ${ }^{73}$ mit dem Ziel einer abstrakten Ordnung des rechtlichen Wissens, sondern ihr geht es um rechtliches Wissen zur Orientierung und Anleitung praktischer Handlungen und Entscheidungen. Die Rechtswissenschaft ist insofern Handlungs- und Entscheidungswissenschaft. ${ }^{74}$

Als solche umfasst sie jedenfalls in Deutschland zunächst die Rechtsdogmatik. ${ }^{75}$ Rechtsdogmatisches Denken ist unmittelbar verknüpft mit dem römisch-rechtlich inspirierten Anspruch auf Systematik und Systembildung, der den meisten anderen Rechtskreisen fremd ist, und verkoppelt auf ganz eigentümliche Weise die Rechts-

68 Vgl. zur Wissenschaftlichkeit als Merkmal europäischer Rechtskultur bereits P. Häberle, Europäische Rechtskultur, Frankfurt am Main 1997, S. 22 ff.; ders., Der europäische Jurist (Fn. 8), S. 27; ders., Der europäische Jurist vor den Aufgaben unserer gemeinsamen Verfassungs-Zukunft - 17 Entwürfe auf dem Prüfstand, JöR n.F. 53 (2005), S. 457 ff. (473); Böttcher, Jurist (Fn. 4), S. 11.

69 Prütting, Folgen (Fn. 4), S. 10.

70 Zur Trennung von Theorie und Praxis in der Juristenausbildung des 18. und 19. Jahrhunderts vgl. Pieroth, Literarische Streifzüge (Fn. 9), S. 797 ff.

71 Möllers/Voßkuble, Staatsrechtswissenschaft (Fn. 41), S. 332.

72 Deutlich H. Dedek, Recht an der Universität: „Wissenschaftlichkeit“ der Juristenausbildung in Nordamerika, JZ 2009, S. 540 (548).

73 A. Hollerbach, Artikel „Rechtswissenschaft“, in: Staatslexikon der Görres-Gesellschaft, Bd. 4, 7. Aufl. 1988, Sp. 751 ff. (751).

74 Vgl. dazu Voßkuble, Methode (Fn. 18), S. 179 ff.

75 Unter „Rechtsdogmatik“ kann eine Klasse von Sätzen (Definitionen, Prinzipien etc.) verstanden werden, die auf das positive Recht und die Rechtsprechung bezogen, aber nicht mit ihrer Beschreibung identisch sind, im Rahmen einer institutionell betriebenen Rechtswissenschaft aufgestellt und diskutiert werden und einen normativen Gehalt besitzen, s. R. Alexy, Theorie der juristischen Argumentation, 3. Aufl. Frankfurt am Main 1996, S. 326. 
praxis mit der Rechtswissenschaft. ${ }^{76}$ Rechtsdogmatik ist aber notwendigerweise ein introvertiertes Konzept. Erst wenn man mithilfe des Nachdenkens über die Grundlagen des Rechts die nationalen Bedingtheiten der eigenen Argumentation erkennt, vermag man auch die vermittelbaren Stärken der eigenen Jurisprudenz auszumachen und zu übersetzen. ${ }^{77}$ Als praktische Wissenschaft erfasst die Rechtswissenschaft deshalb gerade auch diejenigen Disziplinen, die sich mit den philosophischen, gesellschaftlichen und geschichtlichen Grundlagen des Rechts befassen. ${ }^{78}$ Es sind die Grundlagenfächer, die aus der Rechtswissenschaft eine internationale und international anschlussfähige „Gerechtigkeitswissenschaft“ ${ }^{\text {"79 }}$ machen und ihr zugleich das analytische Werkzeug zur Verfügung stellen, um „zu wissen, was sie tut“. Genannt seien hier nur die Rechtsphilosophie und die Rechtsvergleichung. Neu gefragt ist gegenwärtig auch die Rechtssoziologie: Sie erhält im Zuge der Diskussion über das „globale Recht“ neuen Auftrieb, weil sie einen staats- unabhängigen Rechtsbegriff bereitstellt. ${ }^{80}$ Wichtig und schon immer von besonderer Bedeutung ist auch die Rechtsgeschichte. ${ }^{81}$ Insofern halte ich es mit dem großen Rechtsphilosophen Gustav Radbruch, einem engen Freund Hermann Kantorowiczs ${ }^{82}$ :

„Wer nicht das öde Banausentum eines auf die äußerste praktische Notdurft beschränkten Lernbetriebs wünscht, wird einem weiteren Abbau der geschichtlichen Fächer nicht zustimmen können. Gerade eine moderne ökonomisch-soziale Auffassung des Rechts kann durch die geschichtliche Betrachtung nur gefördert werden, wenn diese sich nicht auf Rechtsantiquitäten beschränkt, son-

76 Näher C. Möllers, Methoden, in: Hoffmann-Riem/Schmidt-Aßmann/A. Voßkuhle (Hrsg.), Grundlagen (Fn. 26), $\mathbb{S} 3$, Rn. 35 ff. Allgemein zur Aufgabe der Dogmatik vgl. F. Wieacker, Zur praktischen Leistung der Rechtsdogmatik, in: FS Gadamer, Bd. II, Tübingen 1970, S. 311 ff.; W. Brohm, Die Dogmatik des Verwaltungsrechts vor den Gegenwartsaufgaben der Verwaltung, VVDStRL 30 (1972), S. 245 (246ff.); J. Esser, Dogmatik zwischen Theorie und Praxis, in: FS Raiser, Tübingen 1974, S. 517 ff.; N. Luhmann, Rechtssystem und Rechtsdogmatik, Stuttgart et al. 1974, S. 15 ff.; J. Harenburg, Die Rechtsdogmatik zwischen Wissenschaft und Praxis, Stuttgart 1986, S. 42 ff., 363 ff.

77 Möllers/Voßkuble, Staatsrechtswissenschaft (Fn. 41), S. 331.

78 Hollerbach, Rechtswissenschaft (Fn. 73), Sp. 752. Zur doppelten Rolle der Rechtsphilosophie als Theorie und als praktische Philosophie des Rechts vgl. zuletzt J. Saurer, Das Recht als symbolische Form und Gegenstand der praktischen Philosophie: Zur Rechts- und Staatsphilosophie Ernst Cassirers, ARSP 95 (2009), S. $490 \mathrm{ff}$.

79 Hollerbach, Rechtswissenschaft (Fn. 73), Sp. 751 m. Verweis auf E. Fuchs.

80 Grundlegend G. Teubner, Globale Bukowina. Zur Emergenz eines transnationalen Rechtspluralismus, RJ 15 (1996), S. 255 ff.

81 Vgl. für ein neueres Beispiel einer institutionellen Verbindung von Grundlagenfächern O. Behrends, Vom Sinn der institutionellen Verbindung der drei Grundlagenfächer Rechtsgeschichte, Rechtsphilosophie und Rechtsvergleichung, in: ders./D. von der Pfordten/E. Schumann/C. Wendhorst (Hrsg.), Elementa iuris, Baden-Baden 2009, S. 1 ff.

82 Vgl. Kantorowicz Carter, Gustav Radbruch and Hermann Kantorowicz (Fn. 2). 
dern die ökonomischen und sozialen Triebkräfte der Rechtsentwicklung herausarbeitet." 83

Wer die Grundlagenfächer schleift, gräbt sich daher das frische Wasser der Erkenntnis ab, ohne das jedes juristische Meer zu einem Tümpel wird.

\section{Nachhaltigkeit und Qualität - der europäische Jurist als „Spitzenjurist“}

Damit taucht am Horizont eine weitere Frage auf: Wie lässt sich die Nachhaltigkeit und Qualität der juristischen Ausbildung dauerhaft sichern? Oder anders formuliert: Wie wird der in Deutschland ausgebildete europäische Jurist zum „Spitzenjuristen“?

Bei der Sicherung der Qualität der juristischen Ausbildung in Deutschland geht es nicht nur um das Anliegen, kompetent und professionell im internationalen „Wettbewerb um gute rechtliche Arrangements“ vertreten zu sein. Die Qualität der juristischen Ausbildung ist von grundlegender Bedeutung für das Funktionieren des Rechtssystems, also für Demokratie und Rechtsstaatlichkeit an sich. Der Staat hat deshalb - auch und gerade vor dem Hintergrund der beschriebenen Veränderungsprozesse - eine besondere Verantwortung für die juristische Ausbildung. Es erscheint daher angemessen und notwendig, dass er nicht nur für den unmittelbaren „Staatsdiener" Qualitätsstandards in Gestalt staatlich reglementierter und verantworteter Prüfungen setzt. Auch im Hinblick auf die vielgestaltigen sonstigen Tätigkeitsfelder des europäischen Juristen können wir daher festhalten, dass die gleichbleibend hohe

83 G. Radbruch, Die Universität und die Ausbildung des Juristen, Nachdruck in: D. Strempel (Hrsg.), Juristenausbildung (Fn. 33), S. 263 ff. (265). Zur Auseinandersetzung über die „Geschichte als Lehrmeisterin “ in der Rechtsgeschichtswissenschaft vgl. nur - exemplarisch - D. Klippel, Juristische Zeitgeschichte, Giessen 1985 und M. Stolleis, Aufgaben der Neueren Rechtsgeschichte, oder: hic sunt leones, RJ 4, $251 \mathrm{ff}$. (253 f.). 
Qualität seiner Ausbildung in Deutschland nach wie vor am besten durch eine zentrale Staatsprüfung - das Staatsexamen ${ }^{84}$ - sichergestellt werden kann. ${ }^{85}$

Deutsche Juristen sind nicht von ungefähr für ihre herausragenden Fähigkeiten international bekannt - die breite Spitze von 10-20\%, die das begehrte „Vollbefriedigend" oder besser erreicht, ${ }^{86}$ ist jedenfalls in fachlicher Hinsicht bestens ausgestattet und muss den Vergleich mit den Spitzenabsolventen anderer Ausbildungssysteme keineswegs scheuen. Das ist besonders bemerkenswert, wenn man sich klarmacht, dass jede und jeder die Chance hat, zu diesen besten 10-20 \% zu gehören: Der Weg zum „Spitzenjuristen“ ist nicht wie in den USA an den Zugang zu fast unbezahlbar teuren Elite-Law-Schools gebunden. Er steht jedem offen, der sich der Herausforderung des Staatsexamens stellt. Wir sollten stolz auf diese Errungenschaft des deutschen Sozialstaats sein!

Ich betone die Bedeutung des Staatsexamens für die Sicherung der Qualität der Ausbildung hier aber auch vor dem Hintergrund der aktuellen Diskussion darüber, ob man die juristische Ausbildung aus Anlass der „Bologna-Erklärung“ aus dem Jahr 1999 verbessern könnte. Was ist der Gehalt dieser - völkerrechtlich unverbindlichen - Erklärung? Im Wesentlichen geht es um die Strukturierung des Studiums. Dieses soll zwei Phasen erhalten - eine erste Studienphase, die mit dem Bachelor endet, und im Anschluss daran eine zweite Phase im Sinne eines „Aufbaustudiums“, das mit dem Master abschließt. ${ }^{87}$ Beide Abschlüsse sollen berufsqualifizierend sein, und bei-

84 Zur Geschichte der Staatsprüfung für Juristen, die - nach dem Vorbild des preußischen Systems - 1877 in ganz Deutschland eingeführt wurde vgl. G. Dilcher, Die preußischen Juristen und die Staatsprüfungen: Zur Entwicklung der juristischen Professionalisierung im 18. Jahrhundert, in: K. Kroeschell (Hrsg.), FS Thieme, Sigmaringen 1986, S. 295 ff. (298 ff.); I. Ebert, Die Normierung der juristischen Staatsexamina und des juristischen Vorbereitungsdienstes in Preußen (1849-1934), Berlin 1995; Pieroth, Literarische Streifzüge (Fn. 9), S. $801 \mathrm{ff.}$

85 Für diese Annahme spricht auch die Erfahrung mit der universitären Schwerpunktbereichsprüfung, die seit der letzten Ausbildungsreform gemäß $\mathbb{S}$ S 5-5 d des Deutschen Richtergesetzes (in der Fassung des Änderungsgesetzes vom 11.7.2002, BGBl. I S. 2592, mit den entsprechenden Änderungen der Juristenausbildungsgesetze der Länder) möglich ist. Die ersten Studierenden haben mittlerweile das neue System durchlaufen, und schon beobachten wir den Trend, dass die Noten der universitären Schwerpunktbereichsprüfung im Schnitt deutlich besser, in Einzelfällen sogar "meilenweit" entfernt von der Noten in den schriftlichen Klausuren des staatlichen Teils liegen. Damit will ich der Schwerpunktbereichsprüfung nicht jeden Sinn absprechen; immerhin ermöglicht sie eine stärkere Einbeziehung der Grundlagenfächer in die Examensnote. Gleichwohl ist die Einheitlichkeit und Vergleichbarkeit der Prüfungsstandards nicht in gleicher Weise gegeben, wie im staatlich verantworteten Prüfungsteil. Vgl. zur Problematik bereits H. Schöbel, Die universitäre Schwerpunktbereichsprüfung - "Kuschelnoten ante portas" ?, JA 2008, S. 94 ff. Für einen aktuellen statistischen Überblick vgl. die Ausbildungsstatistik 2008, abrufbar unter http://www.bundesjustizamt.de/cln_115/nn_1634386/DE/Themen/Justizstatistik/Ausbildung/downloads/Ausbildungsstatistik2008, templateId=raw, property=publicationFile.pdf/Ausbildungsstatistik2008.pdf. Daraus ergibt sich, dass im Jahr 2008 im bundesweiten Durchschnitt etwa die Hälfte aller Kandidaten in der universitären Schwerpunktbereichsprüfung die Note "voll befriedigend" oder besser erzielt hat, während dies in der staatlichen Pflichtfachprüfung nur knapp $20 \%$ der Teilnehmer gelungen ist.

86 Im Jahr 2007 waren es bei der ersten Staatsprüfung im Durchschnitt der Bundesländer 16,3 \% (Stand 10. Februar 2009; Statistik abrufbar unter www.bmj.de unter „Statistiken“; Zugriff vom 6.4.2010).

87 Vgl. dazu statt vieler Finkenauer/Rüfner, Einleitung, in: dies./Baldus (Hrsg.), Juristenausbildung (Fn. 4). 
de Abschlüsse sind universitäre Abschlüsse. ${ }^{88}$ Damit sind auch schon die Hauptprobleme benannt, die eine grundsätzliche Vereinbarkeit des Bologna-Systems mit der juristischen Ausbildung in Deutschland sehr zweifelhaft erscheinen lassen. ${ }^{89}$

Noch eine weitere Strukturierungsvorgabe der Bologna-Erklärung ist mit dem von mir skizzierten Leitbild des europäischen Juristen nur schwer kompatibel, nämlich die mit der „Bachelorisierung“ des juristischen Studiums verbundene „Modularisierung“. Eine Modularisierung des Studiums würde letztlich auf ständige studienbegleitende Prüfungen hinauslaufen. Das halte ich nicht für empfehlenswert.

Die juristische Ausbildung als wissenschaftliche Ausbildung sollte keine bloße Fortsetzung der Schule mit anderen Inhalten sein. Ziel und Leitbild des europäischen Juristen muss vielmehr ein nachhaltig aufgebautes rechtliches „know how“ sein. Ein know how, das eine Grundlage bietet für das „lebenslange Lernen“ des europäischen Juristen - auf dass er nicht nur unmittelbar nach dem Staatsexamen, sondern dauerhaft ein „Spitzenjurist“ sein kann.

Der erforderliche Überblick über die Rechtsordnung, Methodenkompetenz und Grundlagenwissen werden durch permanentes Abprüfen kurzfristig erlernten Wissens kaum zu erreichen sein. Die dem Staatsexamen vorausgehende akademische Freiheit des wissenschaftlichen Studiums ist anspruchsvoll, und sie soll anspruchsvoll sein - sie ist Voraussetzung für die Fähigkeit des europäischen Juristen, selbst zu denken und später auf die immer neuen Herausforderungen der Praxis flexibel zu reagieren.

\section{Inter- und Transdisziplinarität - der europäische Jurist im fächerübergreifenden Diskurs}

Das Stichwort „selbst denken“ führt mich zu einem weiteren wichtigen Thema, nämlich der Auseinandersetzung mit anderen Denkstilen und Denkweisen - also mit anderen Disziplinen und anderen Fächern. Der europäische Jurist wird sich in $\mathrm{Zu}$ kunft noch stärker als bisher divergierenden Wissensansprüchen gegenüber sehen, deren Koordinierung auch aus staatlicher Sicht nicht mehr hierarchisch, sondern diskursiv und öffentlich in einem übergreifenden Verhandlungsraum erfolgt, in dem die Akzeptanz von Erklärungen und Lösungen ausgelotet wird..$^{90}$ Es geht also darum, an einem fächerübergreifenden Diskurs teilzunehmen und sich darin zurechtzufin-

88 Wörtlich heißt es in der Bologna-Erklärung vom 19. Juni 1999 (vgl. zu dieser bereits Fn. 6): „Der nach dem ersten Zyklus erworbene Abschluß attestiert eine für den europäischen Arbeitsmarkt relevante Qualifikationsebene. Der zweite Zyklus sollte ... mit dem Master und/oder der Promotion abschließen."

$89 \mathrm{Zu}$ den verschiedenen derzeit kursierenden Modellen vgl. zuletzt Pfeiffer, Probleme (Fn. 6), S. 104 ff.

90 Voßkuble, Expertise (Fn. 41), S. 655. 
den. Der europäische Jurist sollte interdisziplinär ${ }^{91}$ oder wenigstens transdisziplinär arbeiten können. Damit ist nicht gemeint, dass der europäische Jurist auch alle anderen Fächer „können“ soll. Es geht um „praktizierte Interdisziplinarität im Gespräch, nicht im eigenen Kopf “ 92 - es geht um Neugier und darum, sich auf andere Disziplinen einzulassen und sich von ihnen irritieren ${ }^{93}$ und inspirieren zu lassen. Ebenso, wie er sich auf andere Rechtsordnungen, fremde Rechtskonzepte und andere Rechtsideen als die gewohnten einlassen können muss, sollte der europäische Jurist imstande sein, mit anderen Disziplinen in Austausch zu treten. Das gilt nicht nur für den Dialog mit Sozial- und Geisteswissenschaften, sondern auch für die innovationsrelevanten naturwissenschaftlich-technischen Fächer. ${ }^{94}$ Gefordert ist daher zumindest eine „transdisziplinäre Offenheit“ des europäischen Juristen..$^{95}$

\section{VI. „Soft Skills“ und Sozialkompetenz - der europäische Jurist als konflikterfahrener Kommunikator}

Wenn der europäische Jurist sich nicht nur auf andere Disziplinen und andere Rechtsordnungen einlassen können soll, sondern wenn er sich darüber hinaus „als Teil einer europäischen Öffentlichkeit" verstehen soll, ${ }^{96}$ wenn er sich an diese wendet, sie mitbegründet, auf sie einwirkt, sie informiert, sich aus und mit ihr bildet, ${ }^{97}$ dann setzt dies freilich bestimmte kommunikative Fähigkeiten voraus: Der europäische Jurist muss kommunikationsfähig sein und dafür nicht nur - wie bereits dar-

91 Zur erforderlichen Interdisziplinarität und für ein „differenziert-integratives Methodenverständnis“ A. Voßkuble, Neue Verwaltungsrechtswissenschaft, ders./W. Hoffmann-Riem/Schmidt-Aßmann (Hrsg.), Grundlagen (Fn. 26), $\mathbb{1}$, Rn. 39; allgemein zu den Problemen der Interdisziplinarität J. Kocka (Hrsg.), Interdisziplinarität. Praxis - Herausforderung - Ideologie, Frankfurt am Main 1987; G. W. Hunold, Grenzbegehungen. Interdisziplinarität als Wissenschaftsethos, Frankfurt am Main et al. 1995; M. Käbisch/H. Maas/S.Schmidt (Hrsg.), Interdisziplinarität. Chancen, Grenzen, Konzepte, Leipzig 2001.

92 S. Baer, Schlüsselbegriffe, Typen und Leitbilder als Erkenntnismittel und ihr Verhältnis zur Rechtsdogmatik, in. E. Schmidt-Aßmann / W. Hoffmann-Riem (Hrsg.), Methoden (Fn. 8), S. 223 ff. (229).

93 Zur „produktiven Irritation“ im Rahmen eines L.L.M.-Studiums vgl. A. v. Bogdandy, The past and promise of doctrinal constructivism: A strategy for responding to the challenges facing constitutional scholarship in Europa, International Journal of Constitutional Law, Vol. 7, Juli 2009, p. $364 \mathrm{ff}$. (413 f.).

94 Zum Konzept „rechtswissenschaftlicher Innovationsforschung“vgl. W. Hoffmann-Riem, Rechtswissenschaftliche Innovationsforschung - Plädoyer für einen trans- und interdisziplinären Dialog zwischen Rechts- und Technikwissenschaft, in: Technikfolgenabschätzung - Theorie und Praxis, 2003, S. $91 \mathrm{ff}$; ders., Innovationsoffenheit und Innovationsverantwortung durch Recht. Aufgaben rechtswissenschaftlicher Innovationsforschung, AöR 131 (2006), S. 255 ff.

95 Vgl. Hoffmann-Riem, Innovationsoffenheit (Fn. 94), S. 269.

96 Vgl. auch P. Häberle, Der europäische Jurist vor den Aufgaben unserer gemeinsamen VerfassungsZukunft - 17 Entwürfe auf dem Prüfstand, JöR n.F. 53 (2005), S. 457 ff. (469 f.) m.w.N.: „Ein ... Aspekt für Wirken und Werke des europäischen Juristen ist das Werden und z.T. schon Vorhandensein einer europäischen Öffentlichkeit. Sie lässt sich vor allem aus der Kunst und Kultur darstellen, sie stellt sich mehr als nur punktuell auch schon aus dem europäischen (Verfassungs-)Recht her: Die Öffentlichkeit des Europäischen Parlamentes, der Beratenden Versammlung des Europarats, des Wirkens der beiden europäischen Verfassungsgerichte EuGH und EGMR, der Berichte des Bürgerbeauftragten und des Rechnungshofes - all dies setzt europäische Öffentlichkeit teils voraus, teils schafft es sie." 
gelegt - Sprachkompetenz besitzen, sondern auch Urteilskraft, Kritikfähigkeit und Meinungsstärke sowie Verantwortungsbewusstsein, dabei zugleich Toleranz, Einfühlungsvermögen, Bescheidenheit, kulturelle Sensibilität und Kreativität; er muss prozedural - in Verfahren - denken und in Aushandlungsprozessen bestehen können.

Diese Aufzählung enthält allerdings teilweise Charaktereigenschaften, die nur begrenzt im juristischen Lehrplan untergebracht werden können und wohl teilweise nicht unmittelbar „gelehrt“ werden können. Hier gilt es, an die Verantwortung der Ausbilder in Lehre und Praxis zu erinnern. Ihre Aufgabe bleibt es, als Mensch und Person mit allen Schwächen und Vorzügen das Ideal des „europäischen Juristen“ zu vermitteln.

\section{Ausblick}

Lassen Sie mich zusammenfassen: Der europäische Jurist ist (1.) Akteur in nationalen, europäischen und internationalen Normerzeugungsprozessen, (2.) Europäer und Kosmopolit, (3.) Generalist und „Wissensexperte“, (4.) theoretisch und wissenschaftlich ausgebildeter Praktiker, (5.) „Spitzenjurist“, (6.) inter- und vor allem transdisziplinär dialogfähiger sowie (7.) sozialkompetenter Teilnehmer kommunikativer Prozesse.

Diesem Leitbild, dem man sich immer nur annähern kann, das man aber nie ganz erreichen wird, fehlt noch der wichtigste Mosaikstein, den ich zum Schluss hinzufügen möchte: Der europäische Jurist trägt in der sich verändernden, komplexen Welt der Europäisierung, Internationalisierung und Globalisierung entscheidende Verantwortung für Demokratie, Rechtsstaatlichkeit und Menschenrechte - für die Werte also, die Grundlage einer freiheitlichen Gesellschaft und des modernen Verfassungsstaates sind. ${ }^{98}$ Der europäische Jurist - wie wir ihn uns wünschen - ist daher kein Technokrat, sondern Träger und Vermittler der Werte, die die „europäische Rechtskultur" ${ }^{\text {99 }}$ ausmachen. Dass zeigt zugleich: Die Aufgabe, ein guter europäischer Jurist zu werden, endet nicht mit dem Zweiten Staatsexamen. Sie ist uns allen aufgegeben, jeden Tag aufs Neue, jeden Tag mehr!

98 Vgl. auch Böttcher, Jurist (Fn. 4), S. 10 f.: „Bei einer solchen Fragestellung [d.i., der nach der europäischen Rechtskultur] gewinnt die Thematik des europäischen Juristen dann eine andere Dimension. Es geht nicht mehr nur um Ausbildungsinhalte und Ausbildungsgänge, um Zugangsschranken und Selektionsmechanismen, sondern um die Essentials, die Wesensmerkmale des europäischen Juristen, um die Frage nach seiner Identität"; W. Berka, Juristenausbildung - Welche Juristen braucht die Gesellschaft?, in: Österreichische Juristenkommission (Hrsg.), Juristenausbildung: Welche Juristen braucht die Gesellschaft, Wien 2007, S. 9 ff. (16): „Fraglich wird zugleich, ob sich ... überhaupt noch ein verbindliches juristisches Berufsethos finden lässt, wenn dieses mehr sein soll als die hochgradig professionelle, erfolgsorientierte und erfolgshonorierte Wahrnehmung der verschiedenen Interessen, denen der moderne Jurist seine Dienste leiht ..."

99 Grundlegend P. Häberle, Europäische Rechtskultur, Baden-Baden 1994. 